Zuerst ersch. in: Musicae Scientiae 10 (2006), 2, S. 245-263

\title{
Teamgeist im Orchester: Das Zusammenspiel zwischen Flow und Führung
}

\author{
SABINE BOERNER* AND CHRISTIAN FREIHERR VON STREIT** \\ "Faculty of Politics and Management, Univen ity of Konstanz, Germany \\ **Faculty for Educational Psychology, Berlin Free University, Germany
}

\section{- Zusammenfassung}

Der Beitrag untersucht Bedingungen und Effekte eines kooperativen Klimas unter den Musikern eines Symphonieorchesters. Ein kooperatives Klima, d.h. gegenseitige Wertschatzung und Zusammenhalt der Orchestermusiker, verbessert die Zusammenarbeit im Orchester und daher die künstlerische Qualităt der Auffohrungen. Als Bedingungen fûr ein kooperatives Klima im Orchester werden eine hohe intrinsische Motivation der Orchestermusiker und eine transformationale Führung durch den Dirigenten angenommen.

Eine empirische Untersuchung von 208 Musikern aus 22 deutschen Orchestern bestatigt den angenommenen qualitatssteigemden Effekt des kooperativen Klimas unter den Musikern. Darüber hinaus zeigt sich ein Interaktionseffekt von transformationaler Fọhrung des Dirigenten und intrinsischer Motivation der Musiker auf das kooperative Klima im Orchester. Eine Steigerung des kooperativen Klimas im Orchester ist somit nicht durch die isolierte Wirkung einer transformationalen Fûhrung des Dirigenten oder intrinsischer Motivation der Musiker zu erwarten, sondern nur durch ihre Kombination. Aus den Befunden werden Schlussfolgerungen für die Praxis des Musizierens im Orchester gezogen.

\section{EINL.IrTUNG}

Eine hohe künstlerische Leistung eines Orchesters verlangt nicht nur virtuos spielende Musiker, sondern insbesondere eine exakte Synchronisation innerhalb der Instrumentengruppen und zwischen den Instrumentengruppen (Boerner, 2002; Boerner et al., 2004; Rasch, 1979). Daher erscheint insbesondere ein kooperatives Klima, d.h. die gegenseitige Wertschätzung und der Zusammenhalt der Musiker, als erfolgsrelevant. Ein kooperatives Klima fordert einen emotionalen Gleichklang der Musiker, der die Vorausserzung für den musikalischen Gleichklang darstellt (Boerner und v, Streit, 2006). Vor diesem Hintergrund steht in diesem Beitrag die Frage im Zentrum, wodurch ein kooperatives Klima innerhalb eines Orchesters gefordert werden kann. 
Das Klima im Orchester wird zum einen durch die Interaktion der Orchesterminglieder untereinander geprägt. Dabei ist anzunehmen, dass die Weise der Zusammenarbeit von der Motivation der Orchestermusiker beeinflusst wird. In Anlehnung an die Studien zur intrinsischen Motivation im Sinne von Flow. (Csikszentmihalyi, 1997) ist $2 \mathrm{u}$ vermuten, dass diese Art der intrinsischen Morivarion auch im Orchesterbereich relevant ist (Boerner, 2002; Burzik, 2000; Murnighan und Conlon, 1991). In dieser Studie wird untersucht, inwieweit eine hohe intrinsische Motivation der Orchestermusiker ein kooperatives Klima innerhalb der und $z$ wischen den Instrumentengruppen fördert.

Zum zweiten wird das Klima im Orchester durch den Führungsstil des Dirigenten beeinflusst. So gibt es in der Empirie Hinweise darauf, dass speziell ein transformational führender Dirigent die Orchesterleistung positiv beeinflusst (Boerner und v. Streit, 2006). Ein transformationaler Führungsstil zeichnet sich aus durch Charisma des Führenden und durch eine inspirierende Motivation sowie intellekruelle Stimulierung der Gefuhrten (Avolio ot al,, 1999). Erklärt wird die erfolgsförderliche Wirkung eines transformational führenden Dirigenten bisher Wher das Erfordernis einer zentralen Fremdkoordination der Orchestermusiker (Boerner, 2002; Hunt et al., 2004, S. 148). In diesem Beitrag wird als ergänzende Erklärungsmöglichkeit die These untersucht, dass ein transformational führender Dirigent insbesondere ein kooperatives Klima unter den Orchestermusikern fördert und auf diese Weise zu einer Sreigerung der Orchesterleistung beiträgt.

\section{KOOPERATIVES KIMMA UND KONSTLERLSCHE QUALTM}

Die Qualität einer Orchesteraufführung hängt zum einen wesentlich vom technischen und künstlerischen Niveau der beteiligten Musiker ab. Darüber hinaus ist ein entscheidendes Qualititsmerkmal der künstlerischen Leistung eines Orchesters der Grad der Synchronisation aller Musiker (Boerner, 2002; Rasch, 1979), Die Stimmigkeit der Interpretation ist ein zentrales Merkmal für Qualitit einer Aufführung (Dahlhaus, 1973, S. 11). Ein einheitliches Klangbild etwa lisst sich nur dann erreichen, wenn alle beteiligten Musiker z.B. die Intonation (die Tongestaltung) und Dynamik (die Gestaltung der Lautstärke) ihres Spiels exakt synchronisieren (Boerner, 2004). Eine gleichmäßige Steigerung der Lautstärke (crescendo) serzt voraus, dass alle Musiker ihre Lautstärke zur selben Zeit und im selben Maße steigern. Eine zu schnelle oder zu langsame Steigerung der Lautstärke einzelner Musiker würde dagegen das crescendo im Gesamtklang des Orchesters zerstören. (Genaugenommen gilt dies vor allem innerhalb der einzelnen Srimmgnuppen: Aufgrund der Spezifika in der Klangerzeugung der unterschiedlichen Instrumente müssen die unterschiedlichen Stimmgruppen eines Orchester mitunter tats̈̈̈chlich leicht zeitversetzt spielen, um beim Hörer den Eindruck eines synchronisierten Spiels zu erreichen (Rasch, 1979).
Die Koordination des Musizierens lässt sich dabei nicht auf „rechnisch" erscheinende Aspekte wie den gemeinsamen Beginn oder das gemeinsame Ende des Spiels reduzieren. Von wesentlicher Bedeutung fir die Orchesterleistung ist die Koordination im künstlerischen Sinne. Eine gemeinsame Sreigerung der Lautstärke oder eine gemeinsame Artikulation serzen vor allem einen emotionalen Gleichklang der Musiker voraus (Williamon und Davidson, 2002, S. 63). Ein emotionale Gleichklang der Musiker stellt zum einen sicher, dass sich die Beteiligten über die generelle "Richtung ${ }^{\alpha}$ der Interpretation eines Werkes einig sind. Zum anderen erleichtert ein emotionaler Gleichklang die spontane Verständigung der Musiker untereinander, die vor allem über nonverbale Kommunikation geschieht. Erst ein emotionaler Gleichklang der Musiker ermöglicht somit einen musikalischen Gleichklang des Orchesters, der für eine stimmige Interpretation notwendig ist (Boerner, 2002). Entsprechend zeigt sich empirisch, dass Streichquartette, in denen sich die Musiker gut verstehen, erfolgreicher sind als solche, in denen die Musiker einander nicht verstehen (Murnighan und Conlon, 1999, S. 180).

Ein emotionaler Gleichklang der Orchestermusiker stellt sich jedoch nicht von selbst ein. Während Solisten oder kleinere Ensembles durch einige wenige Proben ein gemeinsames Verstăndnis und eine gemeinsame Gefühlslage bei de Interpretation eines Werkes erarbeiten (Williamon und Davidson, 2002), ist dies fur ein Symphonieorchester aufgrund seiner Größe und der Heterogenität der Musiker deutlich schwieriger. Die Musiker der verschiedenen Instrumente bzu. Instrumentengruppen pflegen $z T$. unterschiedliche Selbstverständnisse und fühlen sich häufig stärker ihrer Gruppe zugehörig als dem Orchester. Zudem bestehen Unterschiede in Bezug auf die orchesterinterne Hierarchiestufe und die Bezahlung zwischen Tuttisten (rutti, ital. = alle), Stimmfuhrern und Solisten (Erd, 1987). Weitere Unterschiede bestehen in Bezug auf die jeweilige Dauer der Zugehörigkeit zum Orchester, Alter, Nationalităt und künstlerisches und technisches Niveau der einzelnen Musiker. Nach den Befunden der Forschung über „organizational diversity" kann eine heterogene Zusammensetzung von Teams zwar z.B. für die Kreativität der Gruppe vorteilhaft sein; zugleich können jedoch erhebliche Kooperationsbarrieren entstehen, die sich negativ auf die Gruppenleistung auswirken (Williams und O'Reilly, 1998; Gebert, 2004).

Aus diesem Grund kommt dem kooperativen Klima im Orchester eine entscheidende Bedeutung zu. Unter kooperativem. Klima wird in dieser Untersuchung die gegenseitige Wertschaltzung und der Zusammenhalt der Musiker verstanden. In Anlehnung an das Organisationsklima (Joyce und Slocum, 1990) ist damit ein Konstrukt beschrieben, das auf der Aggregatebene des Orchesters angesiedelt ist; inhaltlich kommt es dem nahe, was man auf der Ebene einer Arbeitsgruppe als "Teamgeist" (Gilson et al., 2002) oder Gruppenklima bezeichnen würde. Mit zunehmender wechselseitiger Wertschătzung und wachsendem Zusammenhalt der Musiker steigt die Wahrscheinlichkeit dafür, dass die Musiker im Zusammenspiel einen emotionalen Gleichklang erreichen und "wie ein Instrument" 
reagieren. Je weniger dagegen die wechselseitige Wertschärzung ausgeprägt ist und je konfliktreicher das Klima, desto geringer ist die Chance, einen emotionalen Gleichklang zu erreichen.

HYPOTHESE 1

Es besteht ein positiver Zusammenhang zwischen einem kooperativen Klima unter den Musitern und der kiinsterischen Qualitär der Orchesterleistung.

\section{KOOPERATIVES KUIMA UND INTRINSISCHE MOTIVATION}

Das Klima im Orchester wird wesentlich durch die Zusammenarbeit der Orchestermitglieder geprägt. Dabei ist anzunehmen, dass die Weise der Orchestermitglieder ger speziell der intrinsischen Motivation der Musiker kommt eine hohe Bedeutung zu (1996, S. 201). Definiert man die intrinsische Motivation im (Allmendinger et al. 19. (1994) über die Teilaspekte „challenge $e^{\alpha}$ (Herausforderung) und enjoyment ${ }^{\text {( }}$ (positive Emotionalität), so entspricht sic weitgehend dem, was Csikszentmihalyi (1997) als Flow bezeichnet. Das Erleben von Flow - das selbstvergessene Aufgehen in der Tätigkeit - erscheint im Kontext von

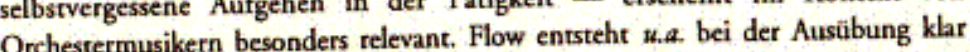
Orchestermusikern besonders relevant. Flow entsteht u.a. bei der Ausubung klar
Trtigkeiten mit unmittelbarer Prozessrückmeldung (Csikrentmihalyi, 1975; Murnighan und Conlon, 1991). So deuten Interviews mit Crilszen-Erlebaisse die Motivation von Orchestermusikern darauf hin, dass Furermusikern zutreffend beschreiben (Burzik, 2000).

Orchestermusikern zutreffend beschreiben (Burzik, 2000). Flow im Sinne vite Sichr der Musiker sowohl die Herausforderung als auch die entspricht. Wenn aus Sicht der Musiker sowohl die Herausforderung als auch die eigenen Fähigkeiten hoch sind, stellt sich Flow ein (Csikszentmihalyi und Lefevre, 1989, S. 816). Das Flow-Erleben selbst konkretisieren Csikszentmihalyi und Lefevre (1989) über unterschiedliche Erlebnis-Qualititen. Die Motivation wird in dem Dimensionenpaar "kognitive Effizienz" ( $z . B$. Konzentration) und "Motivation" ( $z$. Wunsch das zu tun, was man gerade tur) angesprochen. Diese Konzeptionalisierung von Flow wird im Folgenden der Charakterisierung der intrinsischen Motivation im Orchester zugrunde gelegt.

Fin so definierter Flow fördert das kooperative Klima im Orchester. Je mehr sich Sinne der kognitiven Effizienz alle Musiker auf das Gelingen der Aufführun Sin sie bereit, sich auch über den Weg zum Ziel (d.h. die Weise der Interpretation) zu einigen. Aus diesem Grund werden - im Interesse des gemeinsamen Ziels Spannungen zwischen den Musikern tendenziell reduziert und so das kooperative Klima (insbesondere der Zusammenhalt) im Orchester gestärks.
Wenn die Musiker zusärzlich im Sinne der "Motivation“ genau das tun, was sie un möchren, ist zu erwarten, dass ihre gegenseitige Wertschärzung steigt, weil das Zusammensit mir den Kollegen umso mehr als belohnend erlebt wird. Insofer hat das kollektive Flow-Erleben (Sawyer, 2003) selbstbelohnenden Charakter. Die kommt beispielsweise in einem Interview-Zitat mit einem Orchestermusiker zum Ausdruck (Boerner, 2002): „[...] Hier spielen Leure mit ihrer Phanrasic wilde Sau Aus ist eine Freude. [...] und da kommt etwas wieder zusammen, [...] Freve. dieses Miteinander: vier Leute, die mitein musizieren, dieses Gefuhl kann man auch im Orchester haben, das kann man auch musizieren, dieses Gefuhl kefirigenten haben [...]. Wenn man also im Gruppenteam mal, die wilde Sau' rauslassen."

In dem $\mathrm{MaBe}$, wie die Orchestermusiker ihr persönliches Flow-Erleben in denten als Folge der gemeinsamen Anstrengung aller Orchestermusiker deuten, wirkt das Erleben von Flow verstărkend auf das kooperative Klima im deuten, wirkt das Elow Engagement seiner Musikerkollegen zuschreibt, desto mehr steigr seine Wertschätzung für die anderen Musikerkollegen.

HYPOTHESE 2

Das kooperative Klima im Orchester ist umso ausgeprägter, je ausgeprägter di intrinsische Motivation (Flow) der Orchestermusiker ist.

\section{KOOPERTTVES KUMA UND TRUNSFORM aTIONALE FÚHRUNG}

Es wird angenommen, dass das kooperative Klima im Orchester zusätzlich durch den Führungstil des Dirigenten stimuliert wird. In der Literatur gibt es nich den Finweise darauf, dass Dirigenten charismatisch führen (Faulkner, 1973); nur Hinweise darauf, dass Dirigenten charismatisch fuhren (Fus Steigerung der der Führungsstil des Dirigenten trägt auch maßgeblich zur Steigerung der künstlerischen Qualitär des Orchesters bei. So zeigt sich in der Empirie, dass
ein direktiv führender Dirigent, der den Musikern wenig Freiräume gewährt, insbesondere dann die Qualität der Orchesterleistung steigert, wenn er von den Musikern zugleich als fachlich kompetent wahrgenommen wird (Boerner, 2002). Ebenso ist bestätigt, dass ein Dirigent mit hoher Expertenmacht und Identificarionsmacht die künstlerische Qualizät des Orchesters fordert (Krause et al., Identifikationsmacht die künstlerische Qualizat des Orchesters fordert (ransformational führender Dirigent beeinflusst die Orchesterleistung positiv (Boerner und v. Streit, 2006),

Dierles Die transformationale Fuhrung zeichnet Charisma, intellektuelle Stimulierung und inspirierende Motivation. Interpretiert man Führung als eine soziale Intcraktion, so inspire Wird enter kollektiven 
Klimas in Anlehnung an die Theorie der sozialen Identităt im Sinne von Tajfel (1981) plausibilisieren: Personen definieren sich im Sinne der sozialen Identităt w. durch ihre Zugehörigkeit zu einem sozialen Kolleki iv. Diese Selbstkonstruktion kann durch Prozesse der sozialen Interaktion - also auch durch Führung beeinflusst werden. Die transformationale Führungsbeziehung ist dadurch gekennzeichner, dass die Geführten den Führenden idealisieren und sich in hohem Maße mit ihm identifizieren. Je mehr dies der Fall ist, desto mehr sind sie auch bereit, sich für die von ihm propagierten Ziele einzusetzen und Opfer zu bringen (House et al, 1991; Conger, 1989). Auf diese Weise trägt insbesondere das visionărcharismatische Moment der transformationalen Führung zur Ausprägung einer spezifischen gemeinsamen Identität der Gefuihrten bei (Gebert, 2002; Berson et al, 2001; Conger, 1999; Waldmann et al, 2001, S. 135). Eine soziale Identifikation mit der Organisation fördert die Zusammenarbeit und die positive Wertschătzung der Geführten untereinander (Riordan und McFarlane Shore, 1997, S. 344).

In diesem Sinne ist zu vermuten, dass ein transformational führender Dirigent das kooperative Klima im Orchester förders: Der transformational führende Dirigent vermittelt seine künstlerische Konzeption im Sinne einer Vision, die bei den Orchestermusikern hohe Atrrakrivitār besitzt und damit eine kollektive Identitï der Musiker erzeugt. Je mehr dies auf alle Musiker eines Orchesters zurtifft, desto mehr trägt die von allen akzeptierte künstlerische Konzeption des Dirigenten dazu bei, das kooperative Klima der Musiker zu stärken. Die vorstehenden Überlegungen führen zu folgender Hypothese:

HYPOTHESE 3

Das kooperative Klima im Orchester ist umso ausgeprägter, je mehr der Dirigent im Sinne des transformationalen Führungsstils führt.

\section{Methode.}

- Stichprobe. Die vorstehenden drei Hypothesen wurden in einer 2002 und 2003 durchgeführten Befragung von Orchestermusikern untersucht (v. Streit, 2003). Die Fragebögen wurden über die Orchestervorstände an 29 deutsche Symphonieorchester verreilt; der Rücklauf berrug $76 \%$, so dass 22 Orchester in die Untersuchung eingingen. Die untersuchten Orchester harten eine durchschnirtliche Größe von 98 Mitgliedern, wobei pro Orchester maximal 12 Musiker antworteten. Etwa zwei Drittel $(65 \%)$ der Orchester gehören Theatern an, während ein Drittel (35\%) selbständige Symphonieorchester sind. Das Durchschnitrsalter der Befragten liegt bei 40 Jahren $(S D=8,83)$ mit einer durchschnirtlichen Erfahrung als Berufsmusiker im Orchester von 15 Jahren $(S D=9,39) .70 \%$ der Musiker sind mănnlich. In Bezug auf die Instrumentengruppe entspricht die Srichprobe in etwa der tatsilchlichen Zusammensetzung eines Orchesters: 62\% Streicher, 36\% Bläser und 2\% Schlagwerk. Die Mehrzahl der Befragten (64\%) sind Tuttisten, erwa ein Drittel der Musiker (33\%) sind Solisten oder Stimmführer.

- Operationalisierung der Konstrukte. Der Fra wurde in dieser Befragung zum ersten Mal eingeserzt, weil keine Instrumente zur Erfassung der entsprechenden Konstrukte in Orchestern zur Verfügung standen. Auf Vermitrlung der Orchestervorstände wurden die Musiker geberen, die künstlerische Qualität ihres Orchesters, das kooperative Klima, ihre intrinsische Motivation (Flow) und den transformationalen Führungsstil ihres Dirigenten au siebenstufigen Likert-Skalen ( $1=$ gering; $7=$ hoch bzw. $1=$ nein; $7=\mathrm{ja})$ einzuschărzen. Die künstlerische Qualităr wurde pro Orchester von zwei bis drei zusärzlichen Musikern des jeweiligen Orchesters eingestuft (togh unten).

Die interne Konsistenz der hier verwenderen vier Skalen wurde über Cronbachs Alpha (Cronbach, 1951) bestimmt. Die Antworten wurden nicht auf der Individualebene verrechnet, sondern auf Orchesterebene aggregiert. Dabei wurde pro Orchester (und pro Item) ein Mittelwert über die Befragten errechnet, der die Grundlagen für alle nachfolgenden Auswertungen bildet. Die Zulässigkeit der Aggregierung wurde dabei jeweils ubber den $r_{W G(D)}$ - Koeffizienten überprüft James et al., 1984; Koslowski und Hattrup, 1992, S. 162; Bliese, 2000).

Das Konstrukt kooperatives Klima umfasst jeweils vier Items, die die gegenseitige Wertschätzung der Musiker ausdrücken und den Zusammenhal der Musiker untereinander beschreiben. Da beide Aspekte eng miteinander verknüpft sind, wird hier nicht von einer zweifaktoriellen Struktur ausgegangen ( $\mathrm{vg} L$ Tabelle 1, S. 252).

Die transformationale Führung wurde entspreciend der Konzeption von Avolio et al (1999) anhand der drei empirisch relevanten Teilfacerten Charisma, inspirierende Motivation und intellektuelle Stimulierung erhoben. Für jede der drei Facetten wurden zwei ltems formuliert (vol. Tabelle 1), Die intrinsische Motivation der Musiker wurde in Anlehnung an das Konzept von Csikszentmihalyi und Lefevre (1989) anhand der Teilfacetten "cognitive efficiency ${ }^{\alpha}$ und smotivarion" operationalisiert. Für jede der beiden Facetten wurden drei Irems formuliert (tght. Tabelle 1).

Die Messung der künstlerischen Qualităt ist als $\mathrm{Maß}$ für die Leistung eines Orchesters alles andere als unproblematisch (Boerner, 2004). Die meisten in der Literatur vorhandenen Messvorschläge beziehen sich auf einzelne Instrumente. Die Resultate einschlägiger empirischer Studien sind jedoch bisher uneinheitlich, u.a. weil weder über die Kriterien der Bewertung noch über die Weise der Bewertung Konsens besteht (Gabrielsson, 1999, S. 577). Die Vorschlige von Boyle und Radocy (1987), Cooksey (1982) und Sagen (1983) zur Messung der Leistung von Ensembles wie Schulorchestern und -Chören fuhren spezifische Teilaspekte der Auffuthrung wie Tempo, tone control, precision ein. Die Verwendung eines analogen Messinstruments für die kítnstlerische Leistung der hier untersuchten Symphonieorchester hätte eine explizite zusätzliche Experteneinschätzung einer 
Tabelle 1

Operationalisierung der Variablen

\begin{tabular}{|c|c|}
\hline $\begin{array}{l}\text { Koopetatives } \\
\text { Klima } \\
\text { Cronbachs } \\
\text { Alpha }=.90 \\
r_{\text {WG }}=.90\end{array}$ & 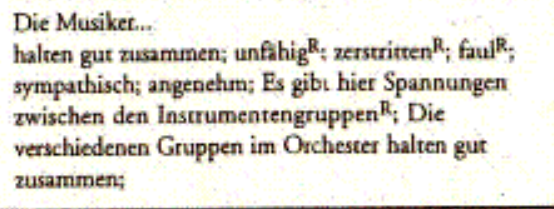 \\
\hline $\begin{array}{l}\text { Trans- } \\
\text { formationale } \\
\text { Fôhrung } \\
\text { des } \\
\text { Dirigenten } \\
\text { Cronbachs } \\
\text { Alpha }=.90 \\
\text { rwow }_{W}=.82\end{array}$ & $\begin{array}{l}\text { Unser Chefdirigent... } \\
\text { hat Charisma; Wir sind stolz, mit ihm zusammen- } \\
\text { zuarbeiten; macht oft volllig neue, uberzeugende } \\
\text { Interpretationsworschlage; erklairt seine Ziele mit } \\
\text { Bildern und Gesten; macht uns klar verständlich, was } \\
\text { et von uns will; ermöglicht uns, bekannre Werke in } \\
\text { einem neuen Licht zu sehen; }\end{array}$ \\
\hline $\begin{array}{l}\text { Intrinsische } \\
\text { Motivation } \\
\text { der Musiker } \\
\text { (Flow) } \\
\text { Cronbachs } \\
\text { Alpha }=.77 \\
r_{W G(t)}=.78\end{array}$ & $\begin{array}{l}\text { Die meisten der Kollegen hier sind mit Leib und } \\
\text { Seele dabei; Es gibr Momente, in denen wir so } \\
\text { konzentriert sind, dass wir alles andere vergessen; } \\
\text { Uns macht das Musizieren hier als solches großen } \\
\text { Spaß; Es kommt vor, dass wir beim Musizieren auch } \\
\text { an anderes denken (z.B. Alltagssorgen); R Es kommt } \\
\text { häufig vor, dass wir uns im Moment der Auffithrung } \\
\text { gegenseitig inspirieren; In manchen Momenten geht } \\
\text { das Spielen wie von selbst. }\end{array}$ \\
\hline $\begin{array}{l}\text { Kuinstlerische } \\
\text { Qualititit des } \\
\text { Orchesters } \\
\text { Cronbachs } \\
\text { Alpha }=.82 \\
r_{W G(D)}=.93\end{array}$ & $\begin{array}{l}\text { Wie wird die kainstlerische Qualinart des Orchesters } \\
\text { won anderen Personen beurteilt (auBerhalb des } \\
\text { Orchesters)? } \\
\text { bezogen auf die Reaktion des Publikums; bezogen auf } \\
\text { die Reaktion anderer Mitwirkender ( } z \text {. Solisten); } \\
\text { berogen auf die Reaktion des Dirigenten; im } \\
\text { Vergleich zu anderen Orchestern. }\end{array}$ \\
\hline
\end{tabular}

Anmerkungen. * Das Item wurde in umgepolter Form verrechnet; Das MaB für die interne Konsistenz der Skala (Cronbachs Alpha) wurde vor der Aggregierung der Daten berechnet. Aus den oben genannten Items wurde pro Skala jeweils ein Skalensummenmittelwert gebildet.

Reihe von Aufführungen pro Orchester erfordert, die im Rahmen der hier vorgestellten Untersuchung jedoch nicht prakrikabel war. Startdessen wurde hier in Anlehnung an frühere Studien (Boerner, 2002; Krause et al., 2002) nicht die künstlerische Qualität selbst zu messen versucht, sondern primär die Reaktion Dritter auf die Leistung des Orchesters. Erbeten wurde nicht die Einschātzung einzelner Auffuhrungen in Bezug auf spezifische Kriterien, sondern die Bewertung der durchschnittlichen Gesamtleistung des jeweiligen Ensembles. Zusätzlich wurde nach der Qualität des Orchesters im Vergleich zu anderen Orchestern derselben Tarifklasse' gefragt (ugl. Tabelle 1). Um zu vermeiden, dass die Höhe der Zusammenhänge durch den „single subject method consistency bias ${ }^{4}$ verfallscht wir (McDonald, 1999), wurde im Gegensarz zu früheren Studien (Boerner, 2002 Krause et al., 2002) hier erstmals die kūnstlerische Qualität des jeweiligen Orchesters nicht von denjenigen Musikern eingestuft, die auch das kooperarive Klima einstuften. Startessen wurden pro Orchester mindestens zwei weitere Musiker geberen, die Qualitär des jeweiligen Orchesters einzustufen.

\section{ERGEBNISSE}

Eine lineare multiple Regression zeigt, dass sich die Qualităt der Orchesterleistung durch das Ausmaß des kooperativen Klimas im Orchester signifikant vorhersagen lässt (vgh. Tabelle 2). Das Ausmaß des kooperativen Klimas im Orchester erklär $41 \%$ der Varianz der Orchesterqualität $(F(2,19)=8.52, p<.050)$. Damit bestätigt sich die Hypothese 1, dass zwischen dem kooperativen Klima unter den Musikern und der künstlerischen Qualitär der Orchesterleistung ein positiver Zusammenhang besteht.

Als Kontrollvariable wurde hier zusätzlich die Tarifklasse der Orchester in die Regressionsgleichung aufgenommen. $\mathrm{Da}$ die Tarifklassen nach der Größe der Orchester eingeteilt werden und zugleich über die Reputation (und die Bezahlung) eines Orchesters Auskunft geben, werden mit dieser Kontrollvariablen inhaldich zwei verschiedene Aspekte erfasst. Die Richtung des Zusammenhangs deutet zwar an, dass eine höhere Tarifklasse mit einer höheren künstlerischen Qualitāi des Orchesters einhergeht. (Da die Variable "Tarifklasse" umgekehrt kodiert ist, bedeuret ein negativer Betawert inhaltlich einen positiven Zusammenhang zwischen Tarifklassenhöhe und künstlerischer Qualität). Die Variable "Tarifklasse ${ }^{\alpha}$ wird jedoch als Prädiktor für die künstlerische Qualität nicht signifikant $(\beta=-.31 ; n$. s.; $N=21$; vgl. Tabelle 2, S. 254).

Der Einfluss der intrinsischen Motivation (Flow) der Musiker und der transformationalen Führung des Dirigenten auf das kooperative Klima im Orchester wurde in einer univariaten Varianzanalyse untersucht (vgl. Tabelle 3). Hierbei zeigen sich zwar signifikante Haupteffekte der intrinsischen Motivation der Musiker $(F=5,65 ; p<.029)$ und der transformationalen Führung des Dirigenten $(F=.7,832$; $p=0.012$ ) auf das kooperative Klima im Orchester. Zugleich zeigt sich jedoch ein Interaktionseffekt zwischen der intrinsischen Motivation der Musiker und der

(1) in Deutschland werden im Prinzip vier Tarifklassen A (hochste Klasse) bis D (niedrigste Klasse) unterschieden. Darüber hinaus exisțieren besondere Tarifverträge, die über der Tarifklasse A liegen. An dieser Untersuchung nahmen Orchester der besonderen Tarifklasse sowie der Klassen A, B und D teil. 
Tabelle 2

Ergebnisse der linearen multiplen Regressionsanalyse: Kooperatives Klima und Tarifklasse als Prädiktoren für die kûnstlerische Qualität des Orchesters

\begin{tabular}{|lc|}
\hline Prïdiktoren & $\begin{array}{c}\text { Kriterien } \\
\text { Künstlerische Qualititit } \\
\beta\end{array}$ \\
\hline Kooperatives Klima & $.49^{* *}$ \\
Tarifklasse & -31 n.s. \\
$R$ & .69 \\
$R$ & $.47(.41)$ \\
$R^{2}\left(R^{2}{ }_{w j}\right)$ & $8.515^{\circ}$ \\
$d f 1, d f 2$ & 2,19 \\
$N$ & 21 \\
\hline
\end{tabular}

Anmerkungen. $B=$ standardisierte Regressionskoeffizienten; $R=$ multiples $R ; R^{2}=$ erklärter Varianzanteil; $R^{2}$ ad $=$ =adjusted $R^{2}, \cdot p<.05, \cdots p<.01, \cdots p<01$; das redurierte $\mathrm{N}$ kommt durch missing values zustande.

transformationalen Führung des Dirigenten auf das kooperative Klima im Orchester $(F=.7,427 ; p=.015)$. Da der Interaktionseffekt signifikant wird, dürfen die Haupteffekte nicht interpretiert werden (Jaccard und Turrisi, 2003, S. 24).

Tabelle 3

Der Einfluss von transformationaler Führung des Dirigenten und der intrinsischen Motivation der Orchestermusiker auf das kooperative Klima im Orchester

\begin{tabular}{|l|rrrrr|}
\hline \multicolumn{1}{|c|}{ Abhăngige Variable: } & SS & ff & MS & $F$ & $p$ \\
kooperatives Klima im Orchester & & & & & \\
\hline Gesamtmodell & 8.83 & 3 & 2.94 & 8.64 & .001 \\
Fehler & 6.13 & 18 & .34 & & \\
Intrinsische Motivation & 1.93 & 1 & 1.93 & 5.65 & .029 \\
Transformationale Führung & 2.67 & 1 & 2.67 & 7.83 & .012 \\
Intrinsische Motivation X & 2.53 & 1 & 2.53 & 7.13 & .014 \\
$\quad$ Transformationale Führung & & & & & \\
\hline
\end{tabular}

Anmerkung, Ergebnisse der univariaten Varianzanalyse

Nach dem vorliegenden Befund lassen sich daher die Hypothesen zwei und drei nicht bestätigen, weil weder die intrinsische Motivation der Musiker noch die transformationale Führung des Dirigenten - jeweils für sich allein betrachtet einen Einfluss auf das kooperative Klima im Orchester haben. Die signifikante
Interakrion zwischen der intrinsischen Motivation der Musiker und der transformationalen Führung des. Dirigenten lässt vielmehr die folgende Interpretation zu: Die intrinsische Motivation der Musiker führt nur dann zu einer Steigerung des kooperativen Klimas im Orchester, wenn zugleich de transformationale Führungsstil des Dirigenten hoch ausgeprägt ist (vgl Abbildung 1).

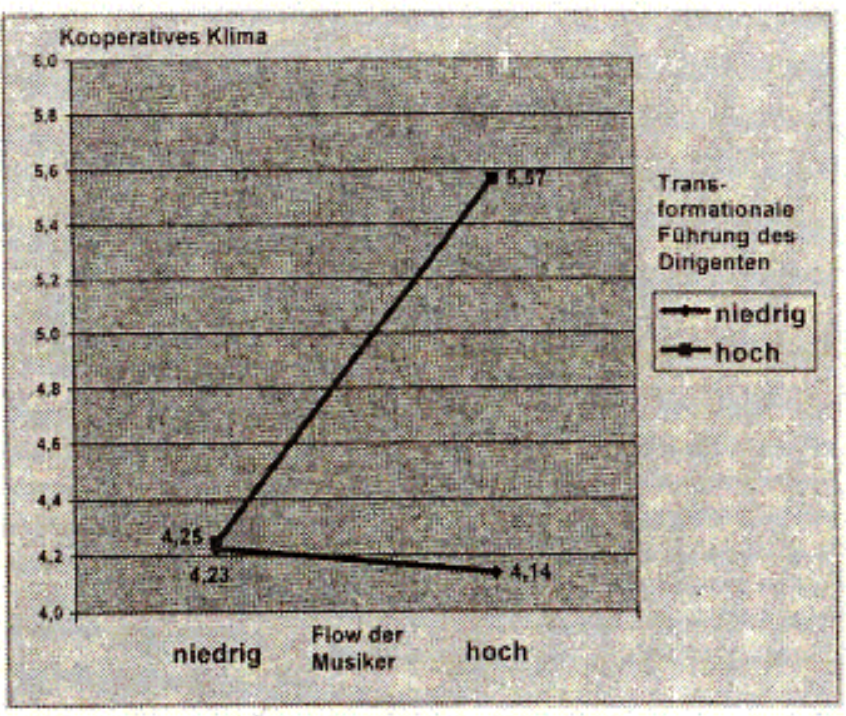

Anmerkungen. Die Variabien wurden am Median gesplittet.

Abbildung 1.

Der Interaktionseffekt zwischen transformationaler führung und Flow auf das kooperative Klima im Orchester aus der Perspektive der transformationalen fühning

Der positive Effekt von Flow unter den Orchestermusikern auf das kooperative Klima im Orchester kommt demnach insbesondere durch die kombinierte Wirkung in Zusammenhang mit intensiver transformationaler Führung durch den Dirigenten zustande. Ist die transformationale Führung durch den Dirigenten niedrig ausgeprägt, lisst sich ein positiver Effekt von Flow auf das kooperative Klima im Orchester dagegen nicht ausmachen (vgl. Abbildung 1); durch ein Mehr an Flow unter den Orchestermusikern verbessert sich in diesem Fall das kooperative Klima also nicht. Ähnliches gilt aus der Perspektive der rransformationalen Führung: Der Effekt der transformationalen Führung des Dirigenten auf das kooperative Klima im Orchester ist dann besonders ausgeprigt, wenn zugleich die intrinsische Motivation (Flow) der Orchestermusiker hoch ausgeprägt ist. Bei niedrigem Flow führt eine 
zunehmende transformationale Führung dagegen nur zu einer geringfügigen Verbesserung des kooperativen Klimas.

\section{Diskussion}

Zunächst ist in methodischer Hinsicht einschrä̀nkend anzumerken, dass ein Längsschnitrdesign, das abhängige und unabhängige Variablen zeitlich versetzt erhebc, aussagekräftiger gewesen wäre als das hier verwendete Querschnitrsdesign. Im Gegensarz zu früheren Studien wurde hier jedoch die Einstufung der künstlerischen Qualităt des Orchesters erstmals aus einer zusärzlichen Quelle gewonnen, womit das Problem des ${ }_{x}$ single subject method consistency bias" (McDonald, 1999) hier umgangen wurde.

$\mathrm{Da}$ die Qualitätsratings jedoch auch in dieser Studie nicht von einer unabhängigen Quelle ( $d . h$, außerhalb des Orchesters) gewonnen wurden, sondern von Musikern des jeweiligen Orchesters, besteht im Prinzip die Gefahr einer systematischen Oberschätzung der Orchesterqualität. Befunde aus der Organisationsforschung lassen jedoch darauf schließen, dass eine Selbsteinschătzung der Leistung durch die Mitarbeiter nicht zwingend besser ausfillt als die Messung mithilfe objektiver Indikatoren (Wall et al, 2004 ). Befunde aus früheren Studien der Verfasser (Boerner und Krause, 2001) deuten darüber hinaus darauf hin, dass Orchestermusiker die Qualität ihres Orchesters in der Regel kritischer einschätzen als Externe.

Die vorliegende Studie untersucht den Zusammenhang zwischen künstlerischer Qualitär im Orchester, kooperativem Klima im Orchester, intrinsischer Motivarion der Musiker und transformationaler Führung durch den Dirigenten. Die Befunde bestätigen die Hypothese (1), dass sich durch das Ausmaß an kooperativem Klima unter den Orchestermusikern die künstlerische Qualität der Orchesterleistung vorhersagen lässt. Ein kooperatives Klima begínstigt einen emotionalen Gleichklang der Musiker, der über eine erleichterte Konsensbildung und Verständigung unter den Musikern den musikalischen Gleichklang und damit die künstlerische Qualität des Orchesters fördert. Darüber hinaus wurde angenommen, dass eine hohe intrinsische Motivation der Musiker (Hypothese 2) und ein hoch ausgeprägter transformationaler Führungsstil des Dirigenten (Hypothese 3) sich positiv auf das kooperative Klima im Orchester auswirken. Beide Hypothesen konnten aufgrund der hier vorgelegten Befunde jedoch nicht bestätigt werden. Vielmehr zeigt sich, dass die Wechselwirkung zwischen intrinsischer Motivation der Musiker und transformationaler Führung des Dirigenten Vorhersagen auf das kooperative Klima im Orchester zulässt.

Ohne eine ausgeprägte transformationale Führung des Dirigenten fuhrt eine Steigerung der intrinsischen Motivation der Musiker dagegen nicht zu einer Steigerung des kooperativen Klimas im Orchester. Umgekehrt gilt: Eine ausgeprägte transformationale Führung des Dirigenten steigert nur dann das kooperative Klima im Orchester, wenn zugleich die intrinsische Motivation der Musiker hoch ausgeprägt ist.

Für die Verbesserung des kooperativen Klimas im Orchester kommt es demnach nicht auf die isolierte Wirkung von intrinsischer Motivation der Musiker ode transformationaler Führung des Dirigenten an; die Steigerung des kooperativen Klimas lasst sich durch die kombinierte Wirkung von transformationaler Führung und intrinsischer Motivation erreichen.

Nach den obigen Öberlegungen könnte dies damit zusammenhängen, dass bei hohem Flow zwar die Konzentration und dit: Motivation der Musiker hoch ausgeprägr sind und damir die Voraussetzungen für die Entstehung von kooperativem Klima günstig sind, weil das Konfliktpotential tendenziell abnimmt. Der (über die etreichte künstlerische Qualität vermitrelte) Belohnungswert der intrinsischen Motivation kann jedoch nicht voll zur Geltung kommen, wenn der transformational fuhrende Dirigent fehlt. Speziell in den hier untersuchten großen Symphonieorchestern, bei denen die Musiker nur in begrenztem Ausmaß Hör- und Sichtkontakt haben, ist daher neben der dezentralen Selbstkoordination der Musiker zugleich eine zentrale Koordination durch einen Dirigenten für die Entstehung eines kooperativen Klimas unverzichtbar (Boerner, 2002; Hunt et al., 2004).

Umgekehrt reicht ein transformationaler Dirigent allein nicht aus, um das kooperative Klima im Orchester sicherzustellen. Dies kann zum einen damit erklär werden, dass ein Orchester ein relativ selbständiger - zumindest in Deutschland auch gewerkschaftlich sehr gut organisierter - Organismus ist, der zu seinem Dirigenten in der Regel ein professionelles, mitunter distanziertes Verhältnis pflegr Vor diesem Hintergrund ist die identitätsbildende Wirkung des Dirigenten generell eingeschränkt. Zum anderen arbeiten Orchester in der Regel mir wechselnden Dirigenten zusammen, so dass die Einflusse der jeweiligen Dirigenten in der Rege nur projektbezogen wirken. Das kooperative Klima im Orchester entwickelt sich daher auch unabhängig von Person und Führungsstil des Dirigenten und auch in Bezug auf nicht-musikalische Fragen sowie bei informellen Anlissen.

Für die Praxis des Musizierens im Orchester zei en die Befunde der vorliegenden Studie erstens, dass der sogenannte Teamgeist im Orchester einen erheblichen Einfluss auf die künstlerische Qualitār des Orchesters hat. Nach unseren Ergebnissen lässt sich die resultierende künstlerische Qualităt eines Orchesters fast zur Halfte $\left(R^{2}=41 \%\right)$ durch das Ausmaß an kooperativem Klima unter den Musikern erklären. Bemühungen um den Zusammenhalt des Ensembles "Orchester" erhalten vor diesem Hintergrund einen bedeutenden Stellenwert für die Qualität eines Orchesters. Weitere entscheidende Einflussfaktoren auf die künstlerische Qualitit des Orchesters, die in dieser Studie jedoch nichr untersuch wurden, sind beispielsweise die Fähigkeiten und Erfahrungen der einzelnen Musiker.

Die Entstehung und Förderung eines kooperativen Klimas im Orchester sollte weder den Musikern noch dem Dirigenten allein uberlassen bleiben. Nach unseren 
Befunden ist ein kooperatives Klima im Orchester das Ergebnis des Zusammenwirkens von hochmotivierten Musikern und einem transformational futhrenden Dirigenten. Auf der Seite der Musiker kommt es daher darauf an, eine hohe intrinsische Motivation zu fördern und auch gerade unter den Bedingungen des Ensemblespiels zu erhalten, also gelegentlich beobachtbaren Tendenzen zum "Dienst nach Vorschrift" mit Nachdruck zu begegnen. Auf der Seite des Dirigenten lautet eine Konsequenz, dass Dirigenten nicht nur musikalisches „Expertenwissen ${ }^{\text {" }}$ mitbringen sollten, sondern auch die Fahigkeit zur transformationalen Führung. Zusătzlich bestätigen die Befunde die Erfahrung professioneller Ensembles, dass Dirigent und Orchester zueinander spassen " müssen. Dies gilt nicht nur in musikalischer Hinsicht im engeren Sinne $(z, B$. in Bezug auf Stil und Interpretationsweise), sondern speziell auch für die soziale Interakrion zwischen Musikern und Dirigenten.

\section{(2) Kontaktadresse: \\ Sabine Boerner \\ Chair for Management, especially Strategy \\ and Leadership \\ Faculty of Politics and Management \\ University of Konstanz \\ Universitätsstr. 10 \\ Germany \\ e-mall: sabine.boernereusui-konstanz.de}

\section{- Literaturnachweis}

Allmendinger, J., Hackman, J. R., \& Lehman, E. V. (1996). Life and Work in Symphony Orchestras. The Musical Quarterily 80, 194-219.

Amabile, T. M., Hill, K. G., Hennessey, B. A., \& Tighe, E. M. (1994). The work preference inventory: Assessing intrinsic and extrinsic motivational orientation. Journal of Personality and Sacial Pochology 66 (5), 950-67.

Avolio, B. J., Bass, B. M., \& Jung, D. I. (1999). Re-examining the components of transformational and transactional leadership using the Multifactor Leadership Questionnaire. Journel of Ocrupational and Organizational Prochologx 72, 441-62

Berson, Y., Shamir, B., Avolio, B. J., \& Popper, M. (2001). The rehationship berween vision strength, leadership style, and context. Leadersbip Quarterly.12, 53-73.

Bliese, R. (2000). Within-group agreement, non-independence, and relizbility. In K. J. Klein and S. W. J. Kozlowski (eds), Multilevel theory research, and methods in organization (pp. 349-81). San Francisco: Jossey-Bass,

Boerner, S. (2002). Fabrungyverhatien und Fubbungserfolg - ein Beitrag aw einer Theorie der Filhrong am Beipiel des Musiktshatern [Leadership behaviour and leadership success contribution to 2 theory of Jeadership taking the example of opera companies. Wiesbaden: Gablet.

Boerner, S. (2004). Artistic quality in an opera company - trowards the development of a concepe Nanprofit Managrement and Leadership, 4(4), 425-36.

Boerner, S., \& Krause, D. (2001). Musik als Berufung - zur Motivation im Orchester: Eine empirische Srudie [Music as a vocation - On the motivation of orchestral musicians An empirical investigation]. Das Oncheter, 2001 (11), 8-11.

Boerner, S., Krause, D., \& Gebert, D. (2004). Leadership and cooperation in an orchestra - An empirical study. Human Rosounce Devolopment International, 7(4), 465-79.

Boerner, S., \& v. Streit, C. (2006). Gruppenstimmung (group mood) als Erfolgsbedingung transformationalet Führung - Engebnisse einer empirischen Untersuchung [Group mood as prerequisite of transformational leadership - Results of an empirical

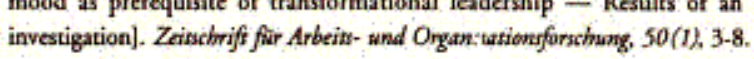

Bortz, J. (1999). Statistrik far Sozishoisenschafiler [Staristics for social scientists]. Berlin: Springer.

Boyle, J. D., \& Radocy, R. E. (1987). Messurement and evalustion of mavical experience. New York Schirmer Bools.

Burzik, A. (2000). Flow-Eifatrungen bei Onchestermusikern: Eine qualitative Studie iber fondernde wnd hemmende Bedingungen [Ochestra musicians' experience of flow: A qualitative study on flow-enhancing and flow-reducing conditions]. Unveroffentlichte Diptomarbeit, Universität Bremen.

Cooksey, J. N. (1982). Developing an objective approach to evaluating music performance. In R. Colwell (ed.), Symposism in Music Edwcation. University of Illinois, Illinois (pp. 197-229).

Conger, J. A. (1989). The charismatic leader: Bebind the mystique of exceptional leadenship. San Francisco: Jossey-Bass.

Conger, J. A. (1999). Charismaric and transformational leadership in organizations: an insider's perspective on these developing streams of research. Lesdership Quarteny) 10 (2), 145 . 60. 
Cronbach, L. J. (1951). Coefficient alpha and the internal structuire of tests. Pychometriks, 16 297-334

Csikszenorithatyi, M. (1975). Beqund boredom and anxiefy. San Franciso: Jossey-Bass

Csiksrentmihalyi, M. (1997). Learning flow: The prochology of engagement with everyday life. New York: Basic Books.

Csikstentmihalyi, M., \& Lefevre, J. (1989), Optimal experience in work and leisure. Journal of Perronaling 56, 815-22.

Dahlhaus, C. (1973). Ober musikalische Wernurteile. [On musical value judgements]. In W. Krüerfeld (ed.), Werpopoblematik der Musikdidakrik (pp. 9-18). Ratingen: Henn.

Erd, R. (1987). Kunst als Arbeit. Organisationsprobleme eines Opernorchesters. Sozialk Wels, 43759.

Faulkner, R. R. (1973). Orchestra in interaction: Some features of communication and authority in an artistic organization. The Sociology Quarterly 14, 147-57.

Gabrielsson, A. (1999). The performance of music. In D, Deutsch (ed.), The Pychology of Mustic (pp, 501-602). San Dicgo: Academic Press

Gebert, D. (2002). Filhrnng und Innowastion [Leadership and innovation]. Seuttgart: Kohlhammer Gebern, D. (2004). Innovation durch Teamarbeit - eine kritiche Bestandraufnahme IInnovation by teamwork - a critical summary]. Sturtgart: Koblhammet.

Gilson, L. L., Shalley, C. E., \& Milne, S. H. (2002). The critical role of ereativity in the relustionship between team processes and performance. Paper presented at the Academy of Management Meeting Denver 2002.

House, R. J., Spangler, W. D., \& Woycke, J. (1991). Personality and charisma in the U.S. presidency: A psychological theory of leader effectivenes. Adminisrative Science Quarserly 36, 364-96.

Hunt, G., Stelluto, G. E., \& Hooijberg, R. (2004). Toward new-wave-organization creativity: Beyond romance and analogy in the relationship berween orchestra-conductor leadership and musician creativity. The Lexdership Quarterts 15, 145-62.

Jaccard, J." \& Turrisi, R. (2003). Interuction effecs in multiple regresion (2nd ed.). Thousand Oaks Sage University Press.

James, L. R., Demaree R. G., \& Wolf, G. (1984). Estimating within-group interrater reliability with and without response bias. Journal of Applied Pychology $69(1), 85-98$.

Joyce, W. F, \& Slocum, J. W. (1990). Strategic contect and organizational climate. In B. Schneider (ed.), Organizational climatr and culture. San Fancisco. Jossey-Bass (pp. 130-50).

Kovlowski, S. W., \& Hattup, K. (1992). A disagreement about within-group agreement: Disentangling issues of consistency versus consensus. Journal of Applied Pychology $77(2), 161-7$.

Krause, D., Boerner, S., Lanwehr, R, \& Nachtigall, C. (2002). Strukrurgleichungsmodelle zut Analyse von Machr und Führungserfolg im Orchester (UISREL-models for the analysis of power and leadership success in the orchestra]. Zeitschrift für Personalpsychologie, 1, 124-37.

McDonald, R. P. (1999). Tost theory A unified treatment. Mahwah: Lawtence Erlbaum.

Murnighan, J. K., \& Conlon, D. E. (1991). The dynamics of intense work groups: A Study of British String Quartets. Administratite Science Quarkering 36, 165-86.

Rasch, R. A. (1979). Synchronization in performed ensemble music. Acustica, 43, 121-31.
Riordan, C. M., \& McFarlane Shore, L. (1997). Demographic Diversiry and employee atritudes: An empinical examination of relational demography within work units. Journal of Applited Aychologx 82, 342-58.

Sagen, D. P. (1983). The development and validation of a university band performance rating scale. Journal of Band Research, 18, 1-11.

Sawyer, R. K. (2003). Group creativisy: Musie, theatre, collaboration. Mahwah, N.J.; Etlbaum.

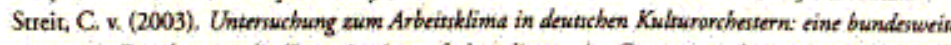
Fragebogenstudie [Investigation of the climate in German orchestras a nationwid empirical study]. Unveroffentlichte Diplomarbei, FU Berlin.

Tajel, H. (1981). Human groups and sacial catogoriex: Studies in social pogchology Cambridge: Cambridge Universiry Press

Waldemann, D. A., Ramires, G. G. \& Housc, R. J. (2001). Does leadership matter? CEO attributes and profitability under conditions of perceived environmental uncertainry. Academy of Monagement Journal, 44 (I), 134-43.

Wall, T. D., Michic, J., Patterson, M., Wood, S. J., Sheehan, M., Clegg, C. W. \& West, M. (2004). On the validity of subjective measures of company performance. Personnel Prychologx. 57, 95-118.

Williamon, A., \& Davideon, J. W. (2002). Exploring co-performer communication. Mustes Scientia, $1(1), 53-72$

Williams, K. Y., \& O'Reilly, C. A. (1998). Demography and diversity in organizations. Reseurch in Organizational Bechavior, 20,77-140. 
- Crear un clima de colaboración en una orquesta: el papel de los músicos y el liderazgo del director

Este trabajo investiga las condiciones previas y los efectos de un clima de cooperacion en una orquesta sinfónica. Un clima de colaboración - es decir, mutua estima y cohesión de los músicos - mejora la cosperación de la orquesta y su calidad interpretativa. La elevada motivación intrinseca de los músicos y ef liderazgo del director son asumidos como pre-requisitos de un clima de cooperación en la orquesta.

El efecto positivo de un clima de colaboración entre his músicos sobre la calidad artistica de la orquesta se ha confirmado con una investigación empirica llevada a cabo con 208 músicos de 22 orquestas sinfónicas aiemanas. Adernass se ha encontrado un efecto de interacción entre la motivación de los músicos y el liderazgo del director en el clima de colaboración en ta orquesta. Es más, no se puede esperar el aumento del clima de colaboración en la orquesta gracias al impacto aislado de la motivación intrínseca o el estilo de liderazgo, sino solamente con la combinación de ambos. Se desarrollan conclusiones prácticas para la interpretación en orquestas sinfónicas.

\section{- Spirito di gruppo in orchestra: il ruolo reciproco di flusso e guida}

Il presente contributo esplora le condizioni e gli effetti di un dima di cooperazione fra i musicisti di un'orchestra sinfonica. Un clima cooperativo, cioé stima reciproca e coesione degli orchestrati, migliora la collaborazione nell'orchestra e di conseguenza la qualita artistica delle esecuzioni. Si assumono come prerequisiti per un clima cooperativo nell'orchestra un'elevata motivazione intrinseca dei muscisti ed uno stile di guida trasformazionale da parte del direttore.

Una ricerca empirica su 208 musicisti di 22 orchestre tedesche conferm Pipotesi di un effetto positivo del dima cooperativo fra gli strumentisti. Si riscontra inoltre un effetto d'interazione sul clima cooperativo dell'orchestra fra la guida trasformazionale del direttore e la motivazione intrinseca dei musicisti. Non ci si puó quindi aspettare un accrescersi del clima cooperativo grazie allinfluenza isolata della guida trasformazionale del direttore, o della motivazione intrinseca de musicisti, ma solo dalla loro combinazione. Da questi risultati si traggono condusion utili per la pratica musicale di un'orchestra sinfonica.

\section{- Création d'un climat de cooperation au sein d'un orchestre : le rôle} du flux des musiciens et le style de direction du chef

On étudie ici a la fois les conditions requises et les effets d'un climat de cooptration au sein d'un orchestre symphonique. Un climat de coopération, autrement dit l'estime mutuelle et la cohesion des musiciens, accroit la cooperation au sein de l'orchestre et, partant, la qualité de Pexécution. Le haut niveau de motivation intrinsèque des musiciens et un style transformationnei de direction du chef sont considérés comme les pré-requis d'un climat de cooperation au sein đ̛un orchestre.

Leffet positif d'un climat de coopération parmi les musiciens sur la qualite artistique de l'orchestre est confirmé par une étude empirique de 208 musiciens de 22 orchestres symphoniques allemands. Qui plus est, la motivation intrinseque des musiciens et le style de direction du chef interagissent sur le climat de cooperation au sein de larchestre: Aussi, un accroissement du dimat de coopération au sein de l'orchestre ne peut-il resulter de l'impact isole de la motivation intrinséque ou du style de direction transformationnel, mais uniquement de leur combinaison. Des condusions pratiques pour l'exécution dans les orchestres symphoniques sont tirées.

- Creating cooperative climate in an orchestra: The role of the musicians' flow and the conductor's leadership style

The paper investigates the preconditions and effects of a cooperative climate in a symphony orchestra. A cooperative climate, i.e., mutual esteem and cohesion of the musicians, enhances the cooperation in the orchestra and hence the performance quality. The musicians' high intrinsic motivation and a transformational leadership style of the conductor are assumed to be prerequisites of a cooperative climate in the orchestra.

The assumed positive effect a cooperative dimate among the musicians has on the artistic quality of the orchestra is confirmed by an empirical investigation of 208 musidians in 22 German symphony orchestras. Moreover, an interaction effect of the musicians' intrinsic mativation and the conductor's leadership style on the cooperative climate in the orchestra is found. Hence, an increase in the cooperative climate of the orchestra cannot be expected from the isolated impact of intrinsic motivation ox transformational leadership style, but only from their combination. Practical condusions for the performance in symphony orchestras are drawn 